\title{
Classifications In Brief: The Paprosky Classification of Femoral Bone Loss
}

\author{
David A. Ibrahim MD, Navin D. Fernando MD
}

Received: 20 May 2016/Accepted: 28 July 2016/Published online: 2 August 2016

(C) The Association of Bone and Joint Surgeons(B) 2016

\section{History}

Projections suggest that the incidence of revision THA performed in the United States will nearly double by 2030 , resulting in as many as 96,700 such procedures per year $[2,18]$. Various indications for hip revision exist, including aseptic loosening, periprosthetic joint infection, fracture, recurrent instability, and more recently, adverse local tissue reactions resulting from metal corrosion. Regardless of the cause of the revision, achieving rigid femoral fixation between the implant and the host bone at the time of revision is essential. The extent of femoral bone loss may represent a substantial impediment to achieving this goal, and as such, having a thoughtful approach to evaluating bone loss is very important.

Various classification systems have been proposed to attempt to address this surgical challenge $[1,7,9,10,12$, $15,19,29]$. One commonly used classification is the Paprosky classification for femoral bone loss, which is a categorization based on bone loss location and degree of severity, and proposes a treatment algorithm for surgical reconstruction based on these measures (Fig. 1) [1, 24].

Each author certifies that he or she has no commercial associations (eg, consultancies, stock ownership, equity interest, patent/licensing arrangements, etc) that might pose a conflict of interest in connection with the submitted article.

All ICMJE Conflict of Interest Forms for authors and Clinical Orthopaedics and Related Research ${ }^{\mathbb{R}}$ editors and board members are on file with the publication and can be viewed on request.

D. A. Ibrahim, N. D. Fernando $(\square)$

Department of Orthopaedic Surgery, Adult Hip and Knee

Reconstruction, University of Washington, 10330 Meridian

Avenue N, Suite 270, Seattle, WA 98133, USA

e-mail: navinf@uw.edu

\section{Purpose}

Revision THA commonly results in some degree of bone loss at the time of implant removal. The most widely accepted standard for femoral revision involves the use of an uncemented femoral component, relying on osteointegration to provide definitive, biologic fixation $[1,17,22,25,37]$. To achieve biologic fixation, a high degree of mechanical stability obtained at the time of femoral reconstruction typically is necessary. Mechanical stability of an uncemented implant is largely dependent on the quality of the residual femoral bone. Many different cementless femoral implants achieve this goal using different approaches, typically accomplished through a combination of implant geometry and surface finish.

A classification system that defines femoral insufficiency based on the location of the bone loss and the likelihood of achieving mechanical and biologic fixation may allow surgeons to plan preoperatively for the type of femoral implant necessary to achieve a durable reconstruction, and this is among the goals of the Paprosky classification. Other purposes of this classification, like so many others, include communication among physicians, categorization of procedures for comparative research, and anticipation of prognosis.

\section{Description}

In the initial paper describing the Paprosky classification, Aribindi et al. [1] categorized femoral bone loss into four types (Table 1). Type I femoral bone loss refers to a defect in which minimal metaphyseal bone loss has occurred, and in which the proximal femoral geometry is maintained. These defects typically are seen after removal of an 
Fig. 1 The Paprosky classification of femoral bone loss, including Type I (I), Type II (II), Type IIIA (IIIA), Type IIIB (IIIB) and Type IV (IV) femoral deficiency, is shown. (Published with the permission of Wolters Kluwer from Della Valle CJ, Paprosky WG. Classification and an algorithmic approach to the reconstruction of femoral deficiency in revision total hip arthroplasty. J Bone Joint Surg Am. 2003;85:1-6.)

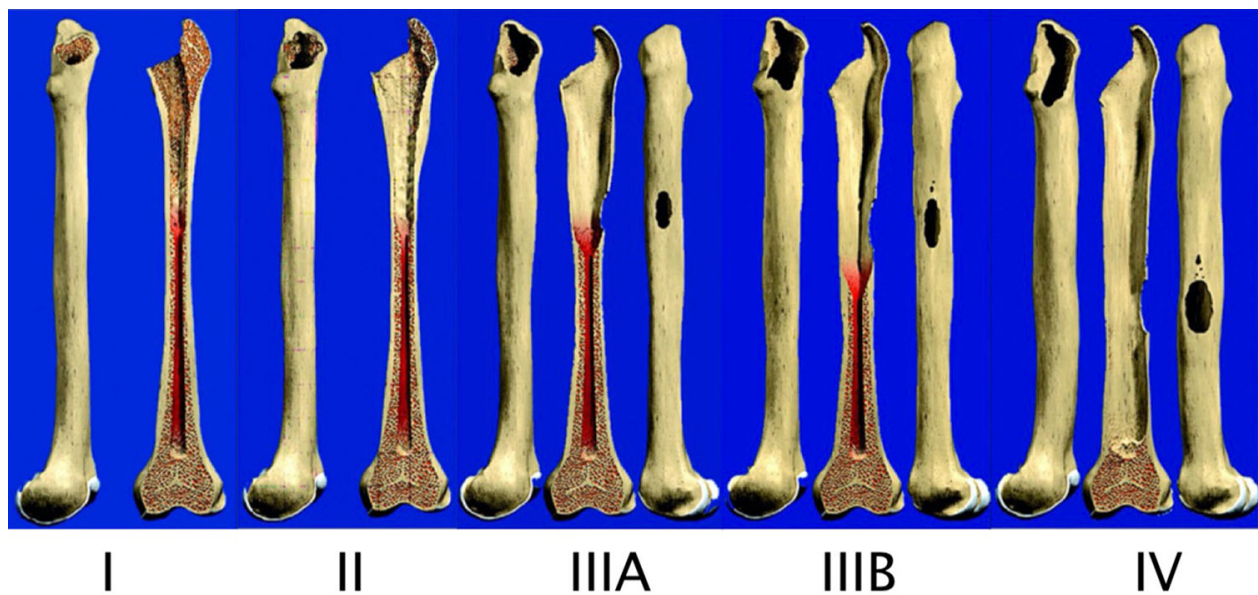

Table 1. Paprosky classification of femoral bone loss

\begin{tabular}{ll}
\hline Type & Description \\
\hline I & Minimal metaphyseal bone loss \\
II & Extensive metaphyseal bone loss, minimal diaphyseal bone loss \\
IIIA & Extensive metaphyseal and diaphyseal bone loss, $\geq 4 \mathrm{~cm}$ intact diaphyseal bone \\
IIIB & Extensive metaphyseal and diaphyseal bone loss, $<4 \mathrm{~cm}$ intact diaphyseal bone \\
IV & Extensive metaphyseal and diaphyseal bone loss, nonsupportive isthmus \\
\hline
\end{tabular}

uncemented implant with a narrow metaphyseal geometry, or removal of an implant with minimal proximal ingrowth potential. These defects can be treated with a cylindrical extensively porous coated stem, although tapered proximally porous coated stems may remain an option if the proximal metaphyseal bone is sufficient to support osteointegration [1, 8, 23, 25, 36, 37]. Although less popular in the United States, other authors also have reported good results with cemented stem revision arthroplasty for cases of preserved proximal femoral geometry [14, 28].

Type II defects refer to femoral bone loss in which the proximal metaphyseal bone has been damaged to a degree where it is may not be mechanically supportive for a proximally fitting implant, nor can proximal biologic fixation be reliably attained in that part of the femur. These defects may be associated with subtle varus remodeling of the proximal femur, although the entirety of the diaphysis remains intact. These defects are commonly seen after removal of a cemented femoral implant, or removal of a proximally fitting stem with a wide femoral geometry. Fixation of a femoral implant that engages the diaphysis, with an ongrowth surface [8, 36] or a porous ingrowth surface [22, 23, 25, 36], typically is recommended, although depending on the degree of metaphyseal bone loss some proximal ingrowth may be expected.
Type III defects are those in which the proximal metaphysis is completely unsupportive, and the endosteal bone is severely deficient or absent. These defects may be caused by grossly, chronically loose femoral stems or revisions performed for posttraumatic total hip conversions. Owing to the absence of a supportive metaphysis, fixation in the diaphysis is necessary. Type III defects are further subcategorized as either Type IIIA $(4 \mathrm{~cm}$ or greater diaphyseal "scratch-fit" [extent of contact between cortical bone and stem] is possible) or Type IIIB (less than $4 \mathrm{~cm}$ diaphyseal scratch-fit is possible because of more-severe bone loss). Based on clinical studies by Paprosky et al. $[22,23]$, use of an extensively porous coated stem when at least $4 \mathrm{~cm}$ of intact diaphyseal bone was present was associated with excellent survivorship (95\% survivorship; mean followup, 13.2 years). Other authors [5, 21] have reported similar results. Conversely, $21 \%$ of patients experienced stem loosening if diaphyseal fixation was less than $4 \mathrm{~cm}$ [23]. Further biomechanical evidence supports that at least 3 to $4 \mathrm{~cm}$ of intact diaphyseal bone is required to resist torsional forces necessary for mechanical stability [20]. If less than $4 \mathrm{~cm}$ of diaphyseal bone is present (Type IIIB), the use of tapered stems (typically splined for rotational stability with an ongrowth surface finish) has been shown to have acceptable clinical survivorship [20]. Many current systems offer modularity, allowing for independent 
diaphyseal and metaphyseal fixation, with substantial intraoperative flexibility for version, limb length, and offset $[6,36]$. Although modularity has potential benefits, fracture at the modular junction and corrosion reactions present challenging complications [6]. Other potential revision options that have shown favorable outcomes in younger and elderly populations with Type III defects include impaction grafting and impaction grafting with cementation [34, 35, 38]. Good outcomes (improved pain and function) with acceptable complication rates also can be achieved in Type III defects with proximal femoral replacement [16, 27, 31].

Paprosky Type IV defects are those with severe metaphyseal and diaphyseal bone loss, typically with severe ectasia (pronounced expansion of endosteal bone with profound cortical thinning) of the femoral canal making uncemented fixation unreliable. Reconstruction options usually are limited to proximal femoral replacements, impaction grafting with a cemented stem, and allograft prostheses composites [16, 27, 31, 34, 35, 38].

\section{Validation}

Brown et al. [4] examined the inter- and intraobserver reliability of the Paprosky classification of femoral bone loss by analyzing a radiologic review done by four arthroplasty surgeons of 205 femurs undergoing revision surgery. The study showed substantial interobserver reliability with a kappa value of 0.61 (95\% CI, 0.55-0.66), and intraobserver reliability with kappa values of 0.81 (95\% CI, 0.76-0.86), 0.78 (95\% CI, 0.72-0.83), 0.76 (95\% CI, 0.70.82 ), and 0.75 (95\% CI, 0.69-0.81), signifying substantial or greater reliability. Their findings suggest that the Paprosky classification system, particularly when analyzed by an experienced surgeon, generally provides a high degree of reliability. They also reported that the reliability of the Paprosky classification was comparable or superior to other established classification systems, such as the Neer classification for proximal humerus fractures, or the Vancouver classification system for periprosthetic hip fractures [3, 32, 33].

Parry et al. [26] reported similar findings in their study in which three orthopaedic surgeons evaluated 23 femurs and classified bone loss based on four separate classification systems (Paprosky, American Academy of Orthopaedic Surgeons, Endo-Klinik, and the novel system proposed by Parry et al.). Kappa values for intraobserver agreement were $0.84,0.6$, and 0.88 for the Paprosky classification, signifying substantial agreement. For interobserver agreement, kappa values ranged from 0.63 to 0.80 , also suggesting substantial agreement and reliability.
Not all authors have been as supportive of the Paprosky classification as Brown et al. [4] and Parry et al. [26]; some have found much lower reliability. One such study by Gozzard et al. [11] had two consultants with a special interest in revision hip surgery and two orthopaedic specialist registrars review 24 femurs to evaluate kappa values for three classification systems, including that of Paprosky. Overall interobserver reliability for the Paprosky system was generally moderate to fair, particularly among the lessexperienced observers. Interobserver reliability overall was 0.42 ( 0.50 for consultants and 0.27 for registrars), and intraobserver reliability was $0.09,0.16,0.47$, and 0.64 for the four readers of radiographs in that study. Unlike other available studies, Gozzard et al. [11] also assessed the validity of the Paprosky classification system by comparing preoperative grades of bone loss with intraoperative assessment of actual bone loss at the time of surgery, finding moderate agreement (kappa score of 0.54). They also reported that the degree of femoral bone loss was underestimated in $12 \%$ of hips studied. Their findings therefore highlight the importance of user experience on reliability, yet still lend some credibility to the validity of the classification system, at least among more-experienced users, and suggest that its validity (that is, its ability to reflect actual surgical findings) is moderate to substantial.

Haddad et al. [13] presented similar findings in their study, in which three adult reconstructive surgeons and three residents reviewed 50 radiographs of failed femoral components with associated bone loss. They found moderate to borderline substantial intraobserver agreement (kappa range, 0.43-0.62) and slight to fair interobserver agreement (kappa range, 0.12-0.29), with no difference in reliability between experts and less-experienced surgeons.

\section{Limitations}

As with any classification system, reliability and validity inevitably will be influenced by various factors, including surgeon experience and adequacy of imaging. This has been shown for the Paprosky classification of femoral bone loss, in which kappa values for inter- and intraobserver reliability have ranged from slight to excellent agreement $[4,11,13,26]$. The aforementioned studies are retrospective and based on radiographic reviews of proximal femoral bone loss. This potentially could lead to underestimation of the extent of femoral bone loss, as the final classification for treatment purposes is based on intraoperative assessment. Nevertheless, the generally good concordance in kappa values in those studies in which arthroplasty-trained surgeons used the Paprosky system, suggests that users may be able to achieve higher reliability and validity with more time and experience $[4,11,26]$. 
The potential limitations of two-dimensional imaging on accurate classification may be a limiting factor to the reliability of the Paprosky classification. Although one might expect less difficulty with characterizing bone loss for long bones in comparison to more complex anatomic structures such as the acetabulum, the limitations of twodimensional imaging on accurate assessments of bone loss have been reported [39]. Gozzard et al. [11] emphasized this concept by describing the mischaracterization of bone loss in more than $10 \%$ of cases.

Two studies [26, 29] challenge the reliability and validity of the Paprosky classification system compared with previous studies [4, 11, 13, 26]. The study by Parry et al. [26] showed good to very good intraobserver reliability with their proposed novel classification system (kappa range, 0.84-1). Mean kappa value for their novel system was 0.87 , compared with 0.77 for the Paprosky classification. The system proposed by Parry et al. [26] achieved equal interobserver reliability as that of the Paprosky classification. In a similar fashion, the new classification system of femoral bone loss proposed by Saleh et al. [29] achieved higher prognostic validity (kappa values of 0.6-0.82) than that of the Paprosky classification in the study by Gozzard et al. [11]. In the study by Saleh et al. [29], three general orthopaedic surgeons, all blinded to each other's ratings, were taught the new classification system proposed by Saleh et al. Radiographic assessments performed by the two surgeons were compared with the intraoperative assessment of bone loss performed by the third surgeon.

To our knowledge, there have been no studies specifically focusing on the role of adjunctive imaging (eg, Judet pelvis views, CT scans) on improving the performance of the Paprosky classification system.

\section{Conclusions}

Femoral reconstruction at the time of total hip revision is challenging and associated with potentially severe complications. The Paprosky classification can help surgeonsparticularly more-experienced surgeons-approach these complex procedures in a thoughtful way, in that it can help guide the implant choices that surgeons make at the time of revision THA [8, 30, 36]. The Paprosky classification offers a simple categorization of proximal femoral bone loss founded on clinical and biomechanical evidence. However, even with such a system, the severity of bone loss can be underestimated. Ensuring optimal preoperative imaging with full-length orthogonal radiographs of the femur is critical to allow for accurate categorization. Despite appropriate preoperative planning, the final categorization of femoral bone loss occurs at the intraoperative setting, with actual femoral deficiencies potentially more severe than expected. As such, surgeons should always be prepared for this possibility, with an armamentarium of implants available to solve more complex problems.

\section{References}

1. Aribindi R, Barba M, Solomon MI, Arp P, Paprosky W. Bypass fixation. Orthop Clin North Am. 1998;29:319-329.

2. Bozic KJ, Kurtz SM, Lau E, Ong K, Vail TP, Berry DJ. The epidemiology of revision total hip arthroplasty in the United States. J Bone Joint Surg Am. 2009;91:128-133.

3. Brady OH, Garbuz DS, Masri BA, Duncan CP. The reliability and validity of the Vancouver classification of femoral fractures after hip replacement. J Arthroplasty. 2000;15:59-62.

4. Brown NM, Foran JR, Valle CJ, Moric M, Sporer SM, Levine BR, Paprosky WG. The inter-observer and intra-observer reliability of the Paprosky femoral bone loss classification system. $J$ Arthroplasty. 2014;29:1482-1484.

5. Chung LH, Wu PK, Chen CF, Chen WM, Chen TH, Liu CL. Extensively porous-coated stems for femoral revision: reliable choice for stem revision in Paprosky femoral type III defects. Orthopedics. 2012;35:e1017-1021.

6. Cross MB, Paprosky WG. Managing femoral bone loss in revision total hip replacement: fluted tapered modular stems. Bone Joint J. 2013;95(11 suppl A):95-97.

7. D'Antonio J, McCarthy JC, Bargar WL, Borden LS, Cappelo WN, Collis DK, Steinberg ME, Wedge JH. Classification of femoral abnormalities in total hip arthroplasty. Clin Orthop Relat Res. 1993;296:133-139.

8. Della Valle CJ, Paprosky WG. The femur in revision total hip arthroplasty evaluation and classification. Clin Orthop Relat Res. 2004;420:55-62.

9. Engelbrecht E, Heinert K. Klassifikation und Behandlungsrichtlinien von Knochensubstanzverlusten bei Revisionsoperationen am Hüftgelenk - mittelfristige Ergebnisse. Primär- und Revisions-Alloarthroplastik Hüft- und Kniegelenk. Berlin, Germany: Springer-Verlag;1987:189-201.

10. Engh CA, Glassman AH. Cementless revision of failed total hip replacement: an update. In: Tullos HS, ed. Instructional Course Lectures. Vol 40. Chicago, IL: American Academy of Orthopaedic Surgeons;1991:189-197.

11. Gozzard C, Blom A, Taylor A, Smith E, Learmonth I. A comparison of the reliability and validity of bone stock loss classification systems used for revision hip surgery. J Arthroplasty. 2003;18:638-642.

12. Gross AE, Allan DG, Lavoie GJ, Oakeshott RD. Revision arthroplasty of the proximal femur using allograft bone. Orthop Clin North Am. 1993;24:705-715.

13. Haddad FS, Masri BA, Garbuz DS, Duncan CP. Femoral bone loss in total hip arthroplasty: classification and preoperative planning. Instr Course Lect. 2000;49:83-96.

14. Haydon CM, Mehin R, Burnett S, Rorabeck CH, Bourne RB, McCalden RW, MacDonald SJ. Revision total hip arthroplasty with use of a cemented femoral component: results at a mean of ten years. J Bone Joint Surg Am. 2004;86:11791185

15. Johnston RC, Fitzgerald RH Jr, Harris WH, Poss R, Muller ME, Sledge CB. Clinical and radiographic evaluation of total hip replacement: a standard system of terminology for reporting results. J Bone Joint Surg Am. 1990;72:161-168.

16. Klein GR, Parvizi J, Rapuri V, Wolf CF, Hozack WJ, Sharkey PF, Purtill JJ. Proximal femoral replacement for the treatment of 
periprosthetic fractures. J Bone Joint Surg Am. 2005;87:17771781.

17. Krishnamurthy AB, MacDonald SJ, Paprosky WG. 5- to 13-year follow-up study on cementless femoral components in revision surgery. J Arthroplasty. 1997;12:839-847.

18. Kurtz S, Ong K, Lau E, Mowat F, Halpern M. Projections of primary and revision hip and knee arthroplasty in the United States from 2005 to 2030. J Bone Joint Surg Am. 2007;89:780785.

19. Mallory TH. Preparation of the proximal femur in cementless total hip revision. Clin Orthop Relat Res. 1988;235:47-60.

20. Meneghini RM, Hallab NJ, Berger RA, Jacobs JJ, Paprosky WG, Rosenberg AG. Stem diameter and rotational stability in revision total hip arthroplasty: a biomechanical analysis. J Orthop Surg Res. 2006;1:5.

21. Moon KH, Kang JS, Lee SH, Jung SR. Revision total hip arthroplasty using an extensively porous coated femoral stem. Clin Orthop Surg. 2009;1:105-109.

22. Paprosky WG, Aribindi R. Hip replacement: treatment of femoral bone loss using distal bypass fixation. Instr Course Lect. 2000;49:119-130.

23. Paprosky WG, Greidanus NV, Antoniou J. Minimum 10-yearresults of extensively porous-coated stems in revision hip arthroplasty. Clin Orthop Relat Res. 1999;369:230-242.

24. Paprosky WG, Lawrence J, Cameron H. Femoral defect classification: clinical application. Orthop Rev. 1990;19(suppl 9):9-17.

25. Paprosky WG, Weeden SH. Extensively porous-coated stems in femoral revision arthroplasty. Orthopedics. 2001;24:871-872.

26. Parry MC, Whitehouse MR, Mehendale SA, Smith LK, Webb JC, Spencer RF, Blom AW. A comparison of the validity and reliability of established bone stock loss classification systems and the proposal of a novel classification system. Hip Int. 2010;20:50-55.

27. Parvizi J, Bender B, Sim F. Revision total hip arthroplasty with femoral bone loss: proximal femoral replacement. In: Wiesel SW, ed. Operative Techniques in Orthopaedic Surgery. Philadelphia, PA: Lippincott Williams \& Wilkins; 2011:823-830.

28. Raut VV, Siney PD, Wroblewski BM. Outcome of revision for mechanical stem failure using the cemented Charnley's stem: a study of 399 cases. J Arthroplasty. 1996;11:405-410.

29. Saleh KJ, Holtzman J, Gafni A, Saleh L, Davis A, Resig S, Gross AE. Reliability and intraoperative validity of preoperative assessment of standardized plain radiographs in predicting bone loss at revision hip surgery. $J$ Bone Joint Surg Am. 2001;83:1040-1046.

30. Sheth NP, Melnic CM, Rozell JC, Paprosky WG. Management of severe femoral bone loss in revision total hip arthroplasty. Orthop Clin N Am. 2015;46:329-342, ix.

31. Shih ST, Wang JW, Hsu CC. Proximal femoral megaprosthesis for failed total hip arthroplasty. Chang Gung Med J. 2007;30: 73-80.

32. Sidor ML, Zuckerman JD, Lyon T, Koval K, Cuomo F, Schoenberg N. The Neer classification system for proximal humeral fractures: an assessment of interobserver reliability and intraobserver reproducibility. $J$ Bone Joint Surg Am. 1993;75:1745-1750.

33. Siebenrock KA, Gerber C. The reproducibility of classification of fractures of the proximal end of the humerus. J Bone Joint Surg Am. 1993;75:1751-1755.

34. Te Stroet MA, Rijnen WH, Gardeniers JW, van Kampen A, Schreurs BW. Satisfying outcomes scores and survivorship achieved with impaction grafting for revision THA in young patients. Clin Orthop Relat Res. 2015;473:3867-3875.

35. te Stroet MA, Rijnen WH, Gardeniers JW, van Kampen A, Schreurs BW. The outcome of femoral component revision arthroplasty with impaction allograft bone grafting and a cemented polished Exeter stem: a prospective cohort study of 208 revision arthroplasties with a mean follow-up of ten years. Bone Joint J. 2015;97:771-779.

36. Valle CJ, Paprosky WG. Classification and an algorithmic approach to the reconstruction of femoral deficiency in revision total hip arthroplasty. J Bone Joint Surg Am. 2003;85(suppl 4): $1-6$.

37. Weeden SH, Paprosky WG. Minimal 11-year follow-up of extensively porous-coated stems in femoral revision total hip arthroplasty. J Arthroplasty. 2002;17(suppl 1):134-137.

38. Wraighte PJ, Howard PW. Femoral impaction bone allografting with an Exeter cemented collarless, polished, tapered stem in revision hip replacement: a mean follow-up of 10.5 years. $J$ Bone Joint Surg Br. 2008;90:1000-1004.

39. Yu R, Hofstaetter JG, Sullivan T, Costi K, Howie DW, Solomon LB. Validity and reliability of the Paprosky acetabular defect classification. Clin Orthop Relat Res. 2013;471:2259-2265. 